\title{
Urban Ecological Sensitivity Evaluation of Anshun, China
}

\author{
Shaolin Huang and Ling Nan
}

\begin{abstract}
Ecological sensitivity analysis is an important basis of city planning. To optimize the land use layout and promote the ecological construction and environmental protection, remote sensing technology and geographic information system technology are used to construct the index system of ecological sensitivity analysis innovatively from five layers of nature. Then the comprehensive analysis and evaluation of the study area are carried out with the spatial distribution of ecological sensitivity. The results show that the ecological sensitivity of the study area is high on the whole. Extremely high and highly sensitive areas account for $\mathbf{3 6 . 0 8 \%}$ of the research region, mildly sensitive and moderately sensitive are as account for $\mathbf{5 2 . 2 0 \%}$, and non-sensitive area is the smallest only account for $11.72 \%$. Existing built-up lands are mainly distributed in the moderately, mildly and non-sensitive areas. However, there are about $20 \%$ of the built-up lands distributed in the extremely high or highly sensitive areas. Overall, the consideration of the ecological sensitivity of new development lands is insufficient. In the later urban expansion, the land development of the extremely high and highly sensitive areas should be strictly limited. Furthermore, ecological restoration should be performed at the same time or in the near future.
\end{abstract}

Index Terms-Ecological sensitivity, remote sensing, geographic information system, spatial distribution, land development.

\section{INTRODUCTION}

Ecological sensitivity refers to the ecological system of natural environmental changes and human disturbance sensitivity [1]-[3]. In the absence of human influence, various ecological processes of ecosystem are maintained with a relatively stable coupling relationship, and the ecosystem is relatively stable. When the interference exceeds a certain extent, some ecological process will show an excessive inflation, leading to serious ecological environment problems (soil and water loss, land desertification, soil salinization, etc.). Then it will destroy the relative stability of the coupling relationship between ecological process and the stability of ecosystem [3]. The essence of the ecological sensitivity assessment is under the clear identification of the status quo in this natural potential, on the basis of ecological environment problems [4], [5].

At present, research on the ecological environment sensitivity analysis at home and abroad mainly focus on the specific single ecological environmental problems, which includes analyzing its sensitivity, evaluating eco

Manuscript received March 5, 2017; revised June 20, 2017.

Shaolin Huang is with the Shenzhen Institute of Building Research, Shenzhen, Guangdong. He is also with Harbin Institute of Technology Shenzhen Graduate School, Shenzhen, Guangdong, China (e-mail: 515742109@qq.com).

Ling Nan is with the Shenzhen Institute of Building Research, Shenzhen, Guangdong, China (e-mail: NanLing@ibrcn.com). environmental problems and obtaining comprehensive ecological sensitivity [6]-[10]. Such as soil sensitivity to acid precipitation, dynamic sensitivity analysis of soil erosion, land degradation and desertification analysis and so on. Comprehensive sensitivity is in the single eco environmental sensitivity analysis conducted on the basis of superposition analysis [11]-[14]. However, the repeated use of one factor leads to excessive enlarging of its factor weights. Soil erosion, desertification, and sensitivity analysis of geological hazards all use slope as a factor, the repeated use of slope factor makes its weight enlarged in the comprehensive index system, which affect the accuracy of the integrated sensitivity analysis results. In consequence, this study takes Anshun City as a study area. From the five layers of nature (lithosphere, pedosphere, biosphere, hydrosphere and atmosphere), this study develops a comprehensive index system without repeating cross impact factor based on the analysis of sensitivity among various spheres. On the basis of further analysis of the integrated ecological sensitivity, the results for the analysis of regional comprehensive ecological sensitivity and the stability of regional ecological system determine the focus of the ecological system protection. It is of great significance to the maintenance of regional ecological security.

\section{METHODS}

The study area is located in the middle of Anshun, China, covering an area of about $2321 \mathrm{~km}^{2}$. It is located in the Yangtze River Drainage Watershed of Wujiang River Basin.

\section{A. Ecological Sensitivity Evaluation Index}

Regional ecological sensitivity is affected by many factors, such as geographic unit, altitude, terrain slope and so on [15]. The evaluation index should be selected according to the actual situation of evaluation target area and evaluation content. Rossi chose the structure characteristics, non-biological risk, endangered animals proportion and isolation as indexes in his research of the ecological sensitivity of protected areas in northern Italy [16]. Xu chose the soil erosion, soil salinization, desertification in the research of evaluating the ecological sensitivity of Fukang [4]. On the basis of field investigation, by considering the ecological environment characteristics of the study area, we finally selected 15 indicators, including geomorphic units, slope, soil organic matter content, as the ecological sensitivity evaluation factors of the research area. According to the influence degree of each evaluation factor of ecological environment, the sensitivity of single ecological factor can be divided into five levels: non-sensitive, mildly sensitive, moderately sensitive, highly sensitive and extremely high sensitive. 
As the sensitivity of each evaluation factor is different, the AHP method is used, i.e., inviting relevant experts to make evaluation of various ecological factors in the relative importance of two factors and concluding the weight of each evaluation factor value, so as to establish the comprehensive evaluation index system of ecological sensitivity in the study area (Table I).

TABLE I: ECOLOGICAL SENSITIVITY ANALYSIS EVALUATION INDEX

\begin{tabular}{|c|c|c|c|c|c|c|c|c|}
\hline $\begin{array}{l}\text { Primary } \\
\text { indicators }\end{array}$ & Weight & Secondary indicators & Weight & Non-sensitive & Mildly sensitive & Moderately sensitive & Highly sensitive & \begin{tabular}{|c|}
$\begin{array}{c}\text { Extremely high } \\
\text { sensitive }\end{array}$ \\
\end{tabular} \\
\hline \multirow{4}{*}{ Lithosphere } & \multirow{4}{*}{0.12} & Geomorphic unit & 0.13 & Basin & $\begin{array}{l}\text { High hills, low } \\
\text { hills }\end{array}$ & Water area & Low mountain & Peak cluster \\
\hline & & Slope & 0.35 & $<8^{\circ}$ & $8^{\circ} \sim 15^{\circ}$ & $15^{\circ} \sim 25^{\circ}$ & $25^{\circ} \sim 35^{\circ}$ & $>35 \%$ \\
\hline & & Mine environment & 0.22 & General & & Generally serious & & Serious \\
\hline & & Geological disaster & 0.30 & Low & & Medium & & High \\
\hline \multirow{3}{*}{ Pedosphere } & \multirow{3}{*}{0.31} & Soil texture & 0.21 & Clay & & Clay loam & & Loam \\
\hline & & Soil pH & 0.22 & $<6$ & & $6 \sim 8$ & & $>8$ \\
\hline & & Soil erosion intensity & 0.30 & Mild & Moderate & Intensity & Very strong & Fierce \\
\hline \multirow{3}{*}{ Biosphere } & \multirow{3}{*}{0.25} & Biological abundance & 0.45 & $<38$ & $38 \sim 49$ & $49 \sim 61$ & $61 \sim 75$ & $>75$ \\
\hline & & Vegetation coverage & 0.35 & $<35 \%$ & $35 \% \sim 50 \%$ & $50 \% \sim 60 \%$ & $60 \% \sim 70 \%$ & $>70 \%$ \\
\hline & & Vegetation type & 0.20 & Rice, wheat & Miscanthus grass & $\begin{array}{c}\text { Sageretia theezans, small } \\
\text { fruit rose bushes }\end{array}$ & $\begin{array}{c}\text { Seguinii white } \\
\text { oak thickets }\end{array}$ & $\begin{array}{c}\text { Pinus } \\
\text { massoniana }\end{array}$ \\
\hline \multirow[b]{2}{*}{ Hydrosphere } & \multirow[b]{2}{*}{0.24} & Gully density & 0.42 & $<2$ & $2 \sim 3$ & $3 \sim 5$ & $5 \sim 7$ & $>7$ \\
\hline & & $\begin{array}{c}\text { Abundance of } \\
\text { underground water }\end{array}$ & 0.58 & Weak & & Medium & & Abundant \\
\hline \multirow[b]{2}{*}{ Atmosphere } & \multirow[b]{2}{*}{0.08} & Sensitivity of acid rain & 0.7 & Non-sensitive & Mildly sensitive & Moderately sensitive & Highly sensitive & \begin{tabular}{|c|}
$\begin{array}{c}\text { Extremely high } \\
\text { sensitive }\end{array}$ \\
\end{tabular} \\
\hline & & Heat island effect & 0.3 & $\begin{array}{l}\text { Medium } \\
\text { temperature, very } \\
\text { high temperature }\end{array}$ & High temperature & Sub-high temperature & $\begin{array}{l}\text { Relatively low } \\
\text { temperature }\end{array}$ & $\begin{array}{c}\text { Extremely low } \\
\text { temperature, low } \\
\text { temperature }\end{array}$ \\
\hline \multicolumn{4}{|c|}{ Evaluation } & 1 & 2 & 3 & 4 & 5 \\
\hline
\end{tabular}

\section{RESUlts AND ANALYSIS}

\section{A. Lithosphere Sensitivity}

Geomorphic unit, slope, mine environment and geological disasters are used to comprehensively analysis the ecological sensitivity of lithospheric. Topography is the foundation of the ecological environment construction. Geomorphological structure results in the formation of terrain, affecting the engineering geology and hydrogeological conditions of different strata. It also makes subsoil bearing capacity of foundation and underground in the water different. Degree of fragile ecological environment system is positively correlated with the gradient, slope is an important terrain factor of ecological sensitivity. Mine environment and geological disasters are the main factors influencing the ecological sensitivity of the study area.

Considering geomorphic unit, terrain slope, mine environmental and geological disasters, coupled with the weight of each factor value, the lithosphere sensitivity of the study area can be deduced using the following formula. The result is shown in Fig. 1.

$$
S S_{j}=\sum_{i=1}^{n} C_{(i, j)} W_{i}
$$

where $S S_{j}$ is the sensitivity index of $j$ space, $C_{(i, j)}$ is sensitivity level value of $i$ factor of $j$ space unit, $W_{i}$ is weight of $i$ factors, $n$ is the number of factors.

\section{B. Pedosphere Sensitivity}

The ecological functions of the soil are mainly biological feed and carrying capacity which directly show the soil nutrient. Land development for the destruction of the soil is difficult to restore, so the higher the value of land ecology, the higher the sensitivity. This research mainly analysis the soil organic matter, soil texture, soil $\mathrm{PH}$ value and soil erosion intensity to analysis the pedosphere sensitivity. Soil organic matter has a very important role in such aspects as soil formation, soil fertility, environment protection and sustainable development of agroforestry. The higher the soil organic matter content, the higher the grade of soil productivity and the higher the ecological value. There is a close relationship among soil texture and soil aeration, protecting fertilizer and water status. Soil PH influences the effectiveness of various elements on the plant by changing the concentration of various ions in soil solution. Soil erosion is the main reason for the degradation of land resources.

Considering soil organic matter, soil texture, soil $\mathrm{PH}$, and soil erosion intensity, as well as coupling with the weight of each factor value, the pedosphere sensitivity of the study area can be deduced using (1). The result is shown in Fig. 2.

\section{Biosphere Sensitivity}

Vegetation coverage is negatively related to the degree of fragile ecological environment. Vegetation is one of the most important parts of the biological resources. It is the most important ecological factor affecting the ecological sensitivity. Biological richness directly decides the whole ecosystem, and it is one of the most important features of the ecological environment quality.

Considering vegetation coverage, vegetation type and 
biological abundance index, as well as coupling with the weight of each factor value, the biosphere sensitivity of the study area can be deduced using (1). The result is shown in Fig. 3.

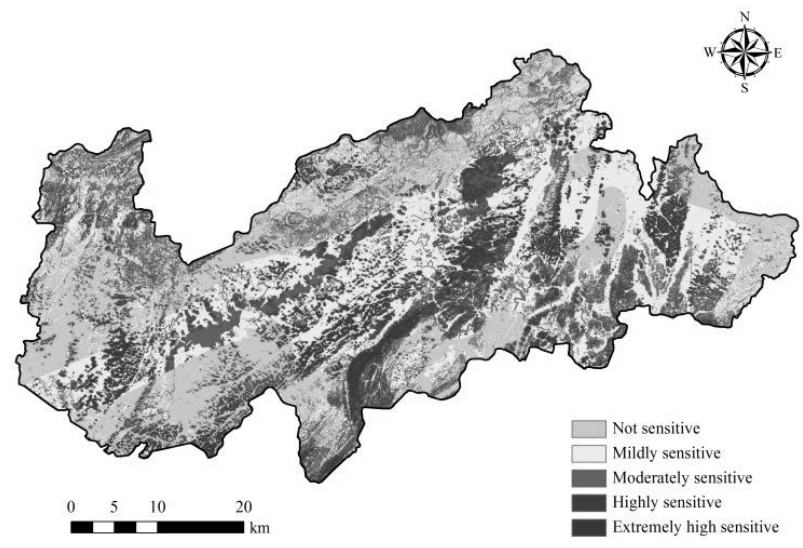

Fig. 1. Lithosphere sensitivity.

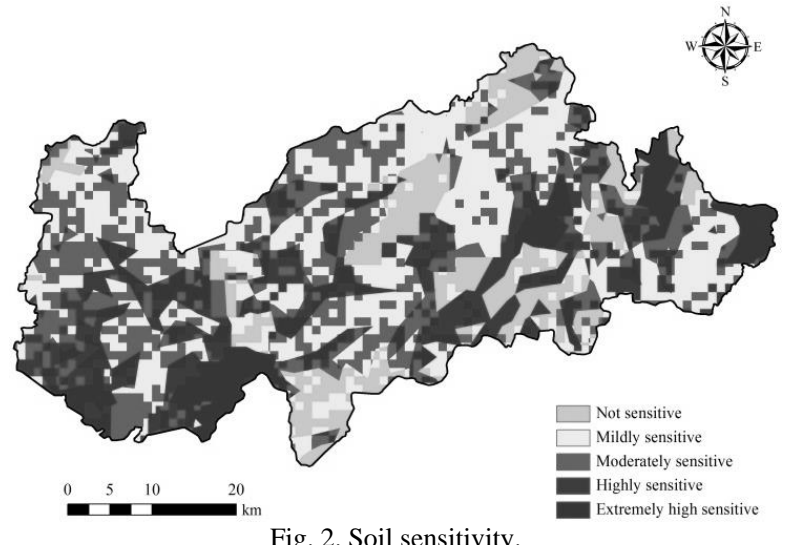

Fig. 2. Soil sensitivity.

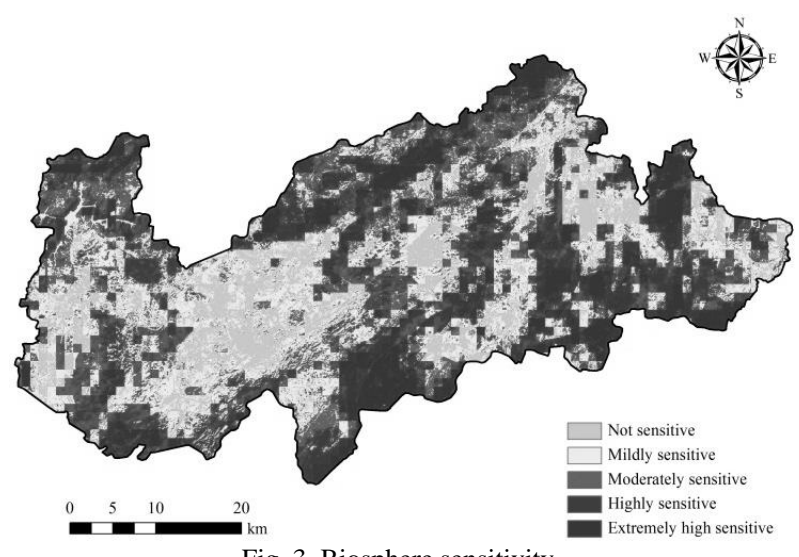

Fig. 3. Biosphere sensitivity.

\section{Hydrosphere Sensitivity}

Hydrosphere sensitivity mainly analyzes the gully density and abundance of underground water. Gully density is the watershed gully erosion degree of evaluation indicators, indicating the development process of valleys. The abundance of underground water is the sign of measuring mining groundwater aquifer when the water yields. Strong rich water aquifer not only provides water for industrial and agricultural development, but also is the important foundation of the improvement of ecological environment in the ecological fragile area.

Considering gully density and abundance of underground water, as well as coupling with the weight of each factor value, the hydrosphere sensitivity of the study area can be deduced using (1). The result is shown in Fig. 4.

\section{E. Atmosphere Sensitivity}

Atmosphere sensitivity mainly uses two indicators to the sensitivity of acid rain and heat island effect. The acid rain is one of the fundamental ecological environment problems in the study area, indirectly affecting the ecological system structure and function of the change of relative ease. Urban heat island effect is caused by the urbanization of a kind of typical climate characteristic, it is a kind of city public nuisance.

Considering the sensitivity of acid rain and heat island effect, as well as coupling with the weight of each factor value, the atmosphere sensitivity of the study area can be deduced using (1). The result is shown in Fig. 5.

\section{F. Comprehensive Sensitivity}

By Combining of each ecological sensitive factor of lithosphere, pedosphere, biosphere, hydrosphere, and atmosphere and stacking the earthquake fault zone, landslide, collapse, ground crack, ground subsidence areas and important rivers water system, the comprehensive ecological sensitivity of the study area can be finally obtained according to formula 2. The result is shown in Fig. 6.

$$
S_{i}=\sum_{i=1}^{n}\left(W_{k} \times C_{i}(k)\right)
$$

where $i$ is the number of spheres, $k$ is the evaluation factor number, $n$ is the number of spheres, $W_{k}$ is the weight of the $\mathrm{k}$ envelops, $C_{i}(k)$ is the sensitivity index value of the $i$ envelops.
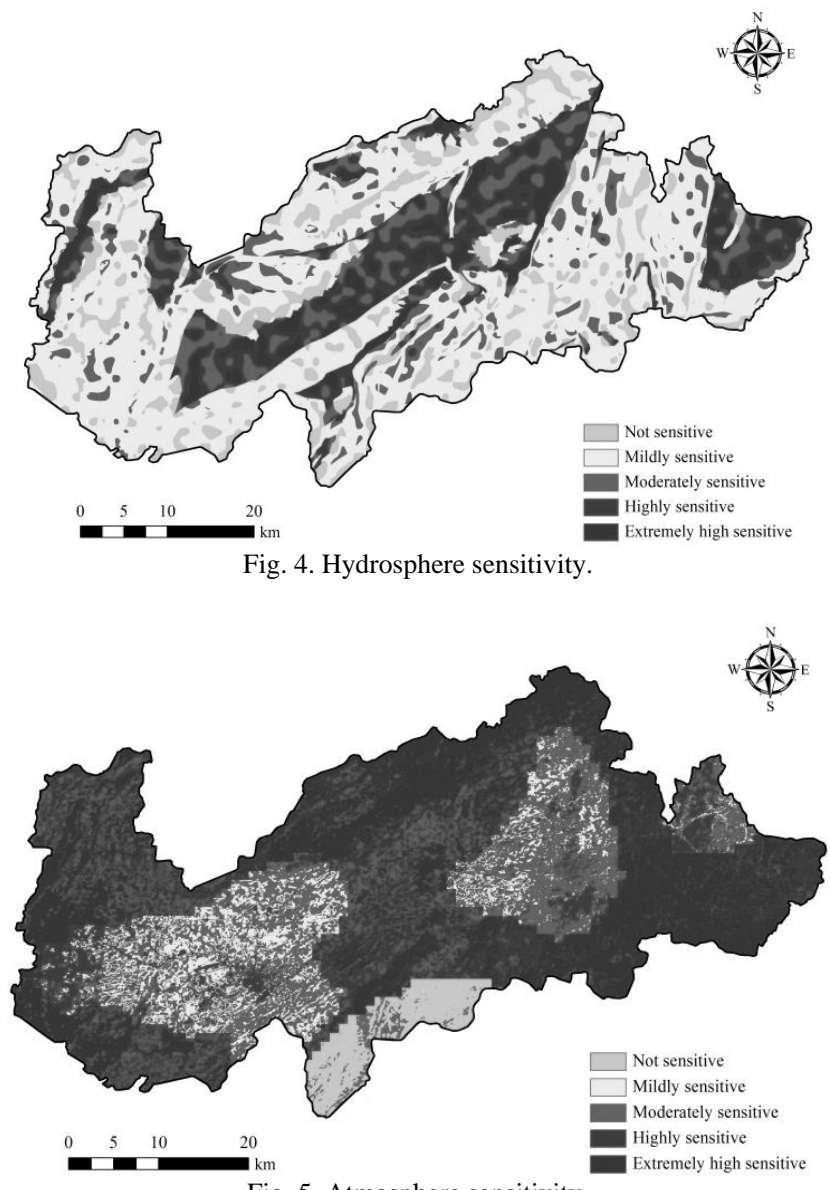

Fig. 5. Atmosphere sensitivity. 


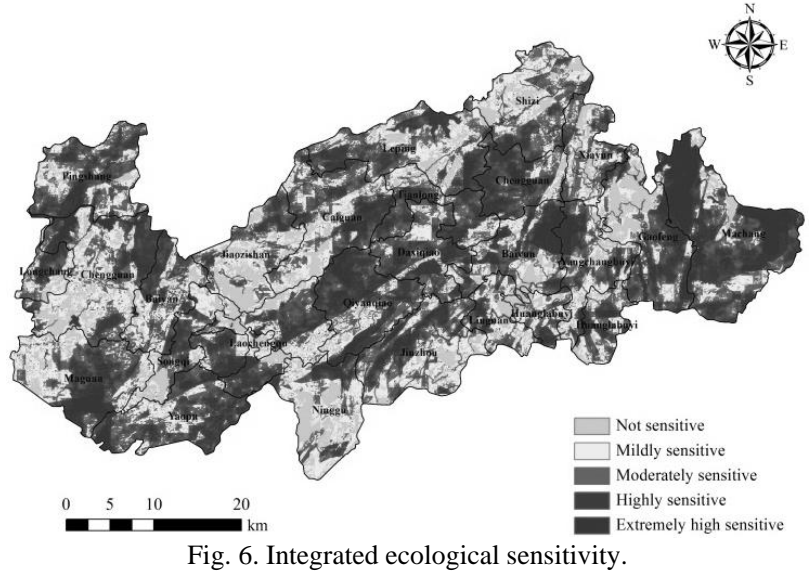

TABLE II: THE SENSITIVITY ANALYSIS OF THE STUDY AREA

\begin{tabular}{|c|c|c|}
\hline Comprehensive sensitivity classification statistics & Area/km & Proportion \\
\hline Non-sensitive & 272.10 & $11.72 \%$ \\
\hline Mildly sensitive & 584.26 & $25.17 \%$ \\
\hline Moderately sensitive & 627.34 & $27.03 \%$ \\
\hline Highly sensitive & 515.78 & $22.22 \%$ \\
\hline Extremely high sensitive & 321.75 & $13.86 \%$ \\
\hline
\end{tabular}

\section{G. Discussion}

After statistical analyzing (Table II), it is found that overall ecological sensitivity of the study area is high. Highly sensitive and extremely high sensitive areas account for $36.08 \%$ of the research region, which are mainly distributed in low peak cluster zone and hongze lake drainage area of Machang Town, Baiyun Town, Daxiqiao town, Qiyanqiao Town, Jiuzhou Town and Maguan Town. Urban planning in the highly sensitive and extremely high sensitive area should be banned or restricted.

Existing built-up lands are mainly distributed in the moderately, mildly and non-sensitive areas. However, there are still about $20 \%$ of the built-up lands distributed in the extremely high and highly sensitive areas. Overall, the consideration of the ecological sensitivity of new development lands is insufficient. In the later urban expansion, the land development of the extremely high and highly sensitive areas should be strictly limited. Furthermore, ecological restoration should be performed at the same time or in the near future.

\section{CONCLUSIONS}

To promote the ecological construction and environmental protection and optimize land use layout, this study constructs the index system of ecological sensitivity analysis without repeat cross impact factors, innovatively from five layers of nature, which provides support for urban planning layout.

Rapid urbanization leads to ecological environment problems. It is necessary to prevent the disorder of urban land expansion, coordinate economic development and ecological environment protection by avoiding use of ecological sensitive areas. The research evaluates the ecological sensitivity of the study area. The results show that the ecological sensitivity of the study area is high on the whole. Extremely high and highly sensitive areas account for $36.08 \%$ of the research region, mildly sensitive and moderately sensitive areas account for $52.20 \%$, while non-sensitive area is the smallest, only account for $11.72 \%$. Existing built-up lands are mainly distributed in the moderately, mildly and non-sensitive areas. However, there are about $20 \%$ of the built-up lands distributed in the extremely high or highly sensitive areas. Overall, the consideration of the ecological sensitivity of new development lands is insufficient. In the later urban expansion, the land development of the extremely high and highly sensitive areas should be strictly limited. Furthermore, ecological restoration should be performed at the same time or in the near future.

\section{ACKNOWLEDGEMENT}

This study was supported by the Shenzhen national and provincial planning project, under contract number GJHS20140903152243522.

\section{REFERENCES}

[1] D. Lebauer, D. Wang, and K. Richter, "Facilitating feedbacks between field measurements and ecosystem models," Ecological Monographs, vol. 83, pp. 133-154, 2013.

[2] Z. Ouyang, X. Wang, and H. Miao, "China's eco-environmental sensitivity and its spatial heterogeneity," Acta Ecologica Sinica, vol. 20, pp. 9-12, 2000.

[3] X. Kang and Y. Liu, "Study on ecological sensitivity evaluation method," Journal of Anhui Agri. Sci., vol. 35, pp. 10569-10570, 2007.

[4] G. Xu, M. Kang, C. Zhao, W. Zhao, and Y. Qin, "Appravisal of eco-sensitivity on Fukang city," Journal of Beijing Normal University (Natural Science), vol. 43, pp. 88-92, 2007.

[5] M. Vardit, G. Gideon, and G. Malka, "Sensitivity analysis for complex ecological models - A new approach," Environmental Modelling and Software, vol. 26, pp. 124-134, 2011.

[6] C. Zhan, X. Song, and J. Xia, "An efficient integrated approach for global sensitivity analysis of hydrological model parameters," Environmental Modelling and Software, vol. 41, pp. 39-52, 2015.

[7] E. Vanuytrecht, D. Raes, and P. Willems, "Global sensitivity analysis of yield output from the water productivity model," Environmental Modelling and Software, vol. 51, pp. 323-332, 2014.

[8] Y. Yang, J. Wang, and B. Yang, "Eco-sensitivity assessment of land in Yunnan Province," Acta Ecologica Sinica, vol. 28, pp. 2253-2258, 2008.

[9] X. Song, X. Li, J. Bai, C. Li, Y. Zheng, and S. Jiao, "The ecological sensitivity evaluation in Yellow River Delta National Natural Reserve," Acta Ecologica Sinica, vol. 29, pp. 4836-4845, 2009.

[10] W. Zhang, J. Wang, X. Ren, J. Gao, and G. He, "GIS-based ecological sensitivity analysis of mountainous areas," Research of Soil and Water Conservation, vol. 20, pp. 44-48, 2013.

[11] X. Song, J. Zhang, and C. Zhan, "Global sensitivity analysis in hydrological modeling: Review of concepts, methods, theoretical framework, and applications," Journal of Hydrology, vol. 523, pp. 739-757, 2015.

[12] D. Li, Z. Gao, X. Fu, X. Wu, and G. Wu, "Characteristic of ecological sensitivity in Yunnan ecological zones," Acta Ecologica Sinica, vol. 30, pp. 138-145,2010.

[13] Z. Liu, Z. Zhou, and B. Guo, "Ecological sensitivity evaluation of significant eco-function areas in Guizhou Province," Ecological Science, vol. 33, pp. 1135-1141, 2014.

[14] X. Xiao, A. Lan, and K. Xiong, "Analysis on ecological environmental sensitivity of Xinjiang River Basin based on GIS," Yangtze River, vol. 46, pp. 68-73, 2015.

[15] H. Yin, J. Xu, C. Chen, and F. Kong, "GIS-based ecological sensitivity analysis in the east of Wujiang City," Scientia Geographica Sinica, vol. 26, pp. 64-69, 2006. 
[16] P. Rossi, A. Pecci, and V. Amadio, "Coupling indicators of ecological value and ecological sensitivity with indicators of demographic pressure in the demarcation of new areas to be protected: The case of the OltrepòPavese and the Ligurian-Emilian Apennine area (Italy)," Landscape and Urban Planning, vol. 85, pp. 12-26, 2008.

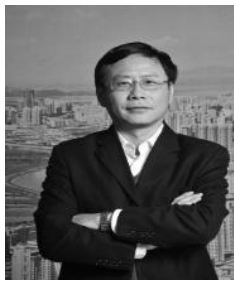

Shaolin Huang received the Ph.D. degree in remote sensing from Fuzhou University, Fuzhou, China, in 2015.

He is currently a post doctor of urban and rural planning at Shenzhen institute of building research. His research interests include remote sensing of environment and resources.

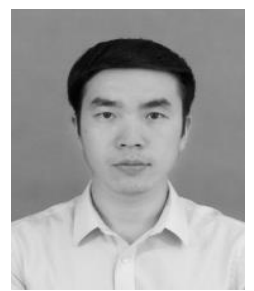

Ling Ning received the Ph.D. degree in geomorphology and quaternary geology from Peking University in 1998.

$\mathrm{He}$ is currently the chief engineer at Shenzhen institute of building research. His research interests include ecological diagnosis of urban and urban planning.. 